\title{
Contribution of Sodium Metasilicate to the Diffusion of Mn in Steel under Tribological
}

\section{Contact at High Temperatures}

Long Wang, ${ }^{1}$ Anh Kiet Tieu, ${ }^{* 1}$ Hongtao Zhu, ${ }^{* 1}$ Shaogang Cui, ${ }^{2}$ Guanyu Deng, ${ }^{1}$ Guojuan Hai, ${ }^{3}$ Jun Yang ${ }^{4}$

1: Faculty of Engineering and Information Sciences, University of Wollongong, Northfields Avenue, Wollongong, NSW 2522, Australia

2: Institute of Advanced Wear \& Corrosion Resistant and Functional Materials, Jinan University, Guangzhou, 510632, China

3: School of Materials Science \& Engineering, Shaanxi University of Science and Technology, Xi'an, 710021, China

4: State Key laboratory of solid lubrication, Lanzhou Institute of Chemical Physics, Chinese Academy of Sciences, Lanzhou 730000, China

Corresponding Author

*(Anh Kiet Tieu) E-mail: ktieu@uow.edu.au; + 61242213061

*(Hongtao Zhu) E-mail: hongtao@uow.edu.au; +61 242214549

Postal Address: Northfield Ave, Wollongong, NSW 2522 Australia 


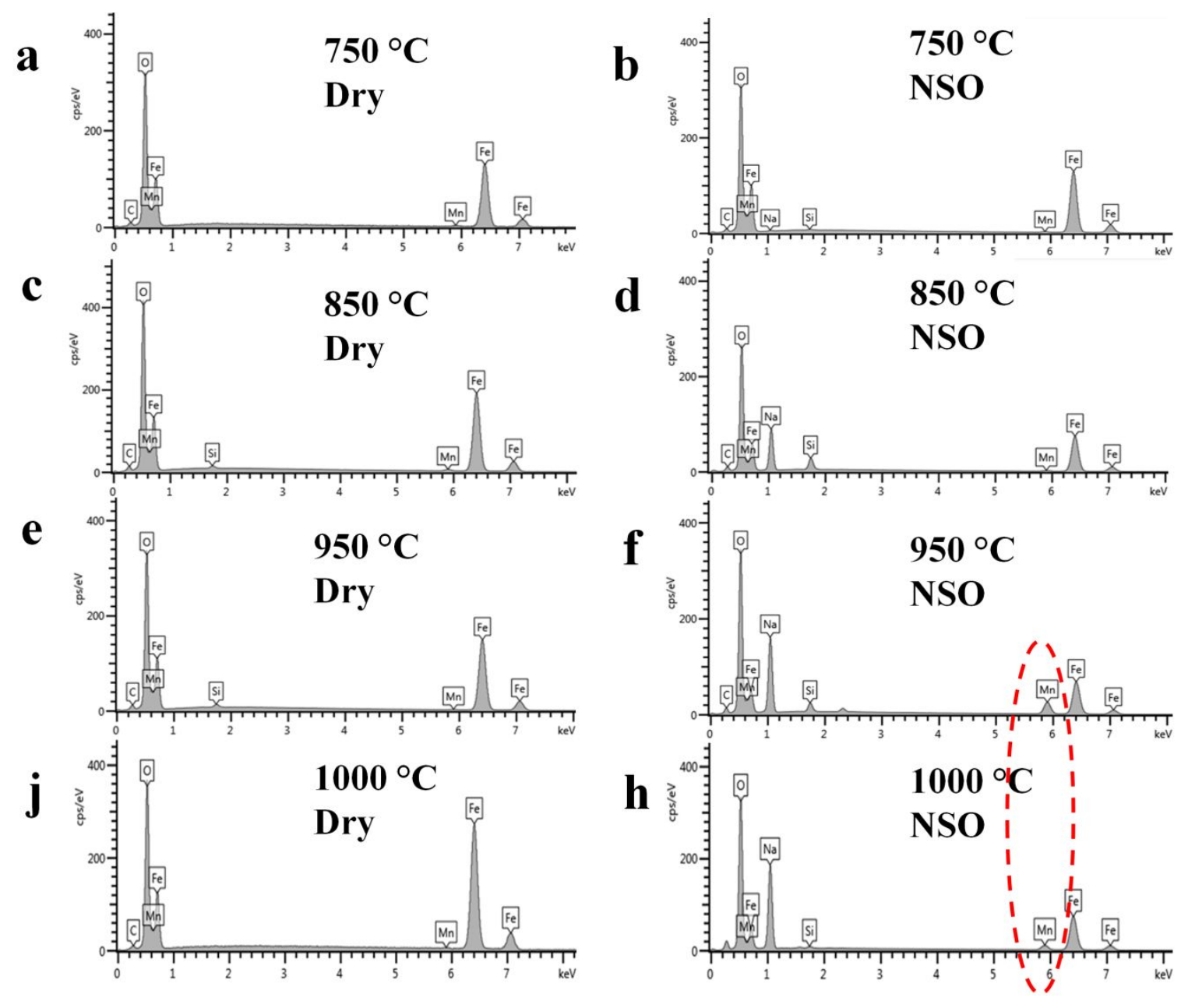

Fig. S1: EDS spectrum of the related wear track surface on the disc tested at different temperature and lubrication conditions. The peaks of $\mathrm{Mn}, \mathrm{Si}, \mathrm{Na}, \mathrm{O}$ and Fe will definitely identify the existence of these elements and further confirm the analysis results of the EDS mapping, the peak of Mn in Fig. S1f and Fig S1h (related to the test result at $950{ }^{\circ} \mathrm{C}$ and 1000 ${ }^{\circ} \mathrm{C}$ ) are obviously higher than other conditions, which is consistent with the mapping results. 
As can be seen, in Fig. S2 only $\mathrm{Na}$, Si and $\mathrm{O}$ signal are observed on the powder surface, the position of the Mn peak in the spectrum is blank, which suggests the absence of Mn in the commercial powder. Similar analysis was also conducted on the powder after heat treatment at $600{ }^{\circ} \mathrm{C}$. The results in Fig. S3 also show the absence of Mn peak at inherent Mn position. Therefore, it can be concluded that the melted sodium metasilicate contributes to the diffusion of Mn from the mild steel.
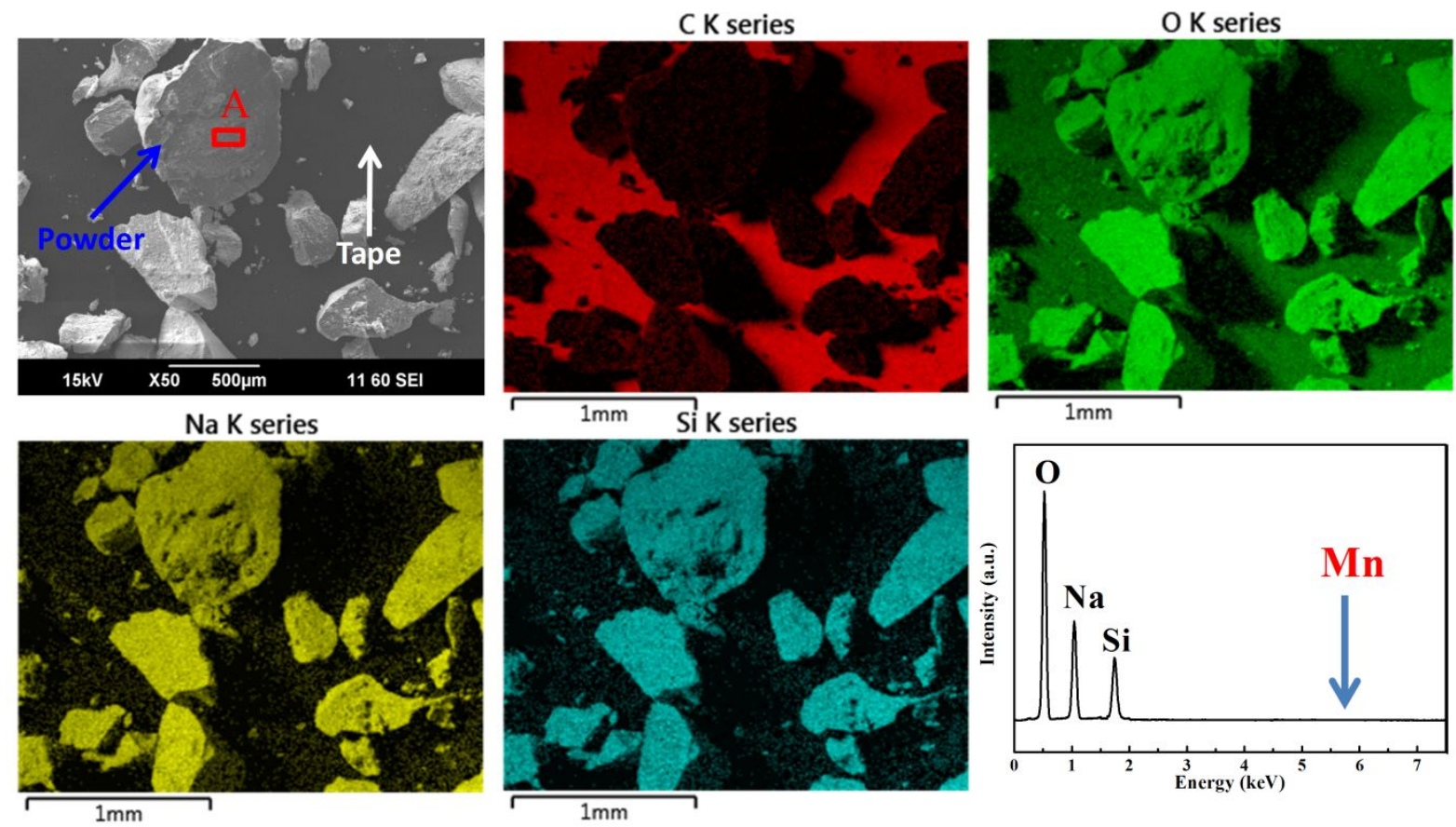

Fig. S2 SEM microscope, EDS mapping and the related EDS spectrum of the commercial sodium metasilicate 

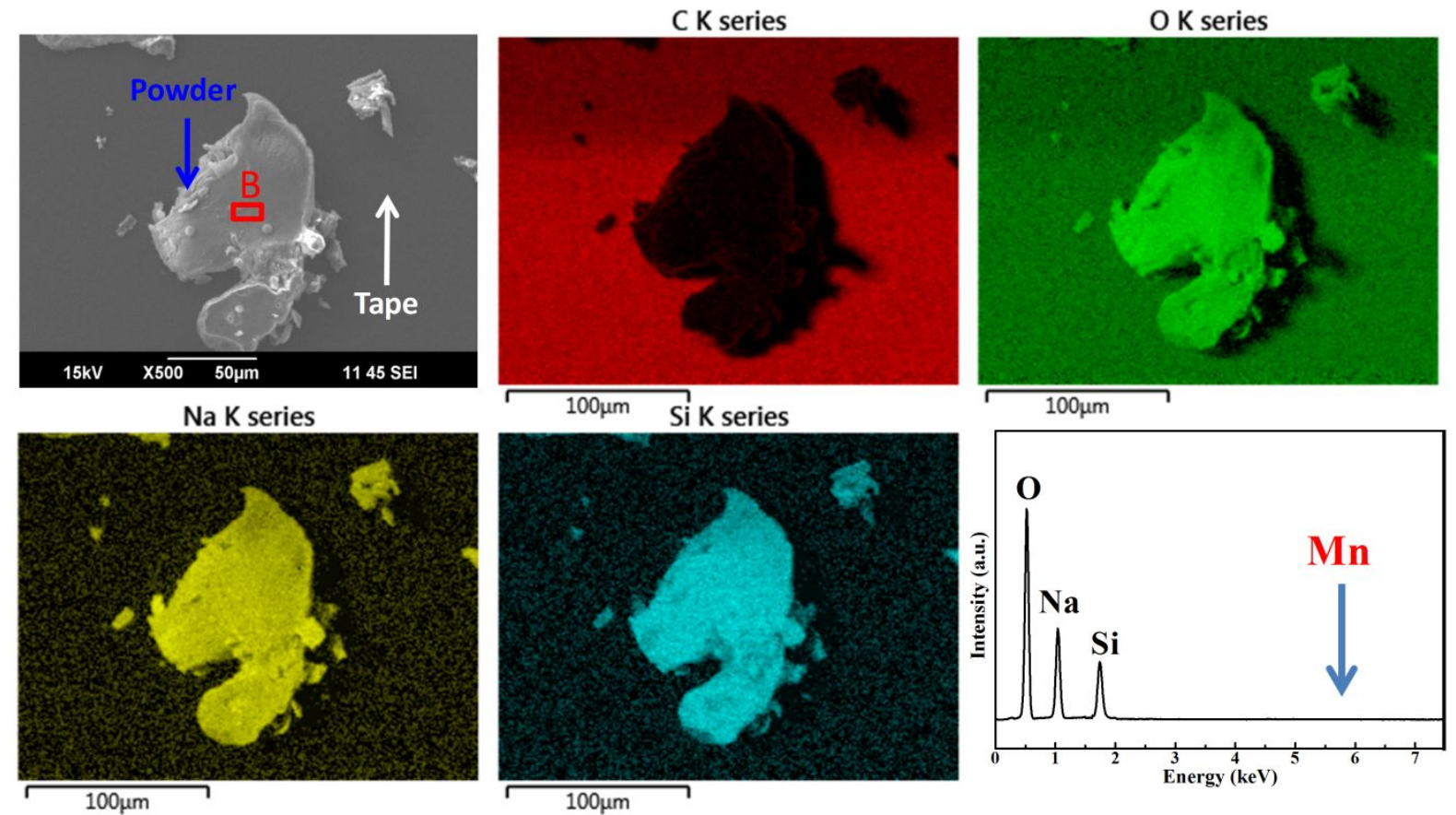

Fig. S3 SEM microscope, EDS mapping and the related EDS spectrum of the commercial sodium metasilicate
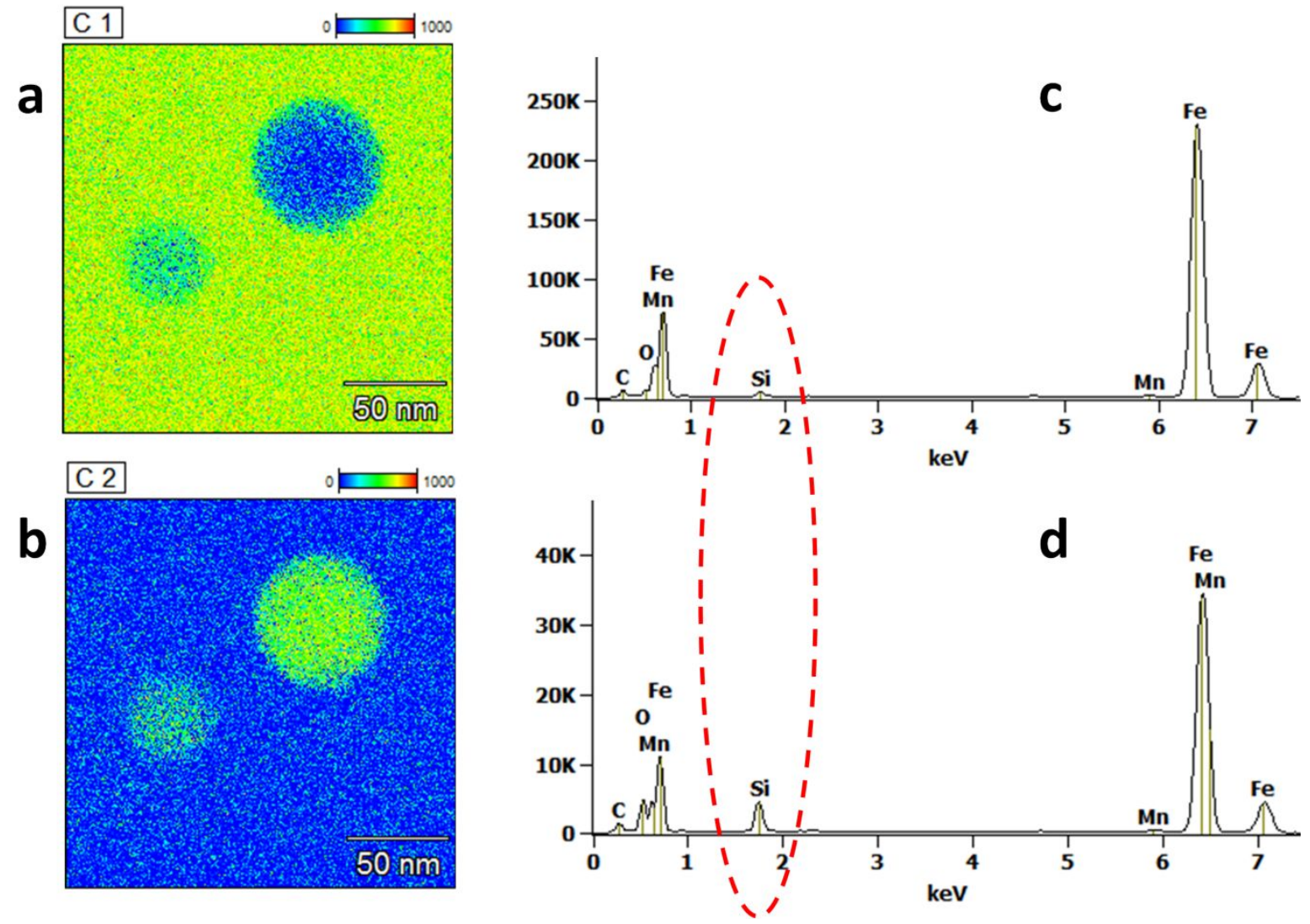
Fig. S4 EDS phase mapping and spectrum of the silica spots area

Fig. S4 shows the EDS phase mapping of the spots area, the blue colour in figure is the background, phase 1 (Fig.S4a) is the steel base, with a strong Fe can be found in its related spectrum in Fig. S4c. Phase 2 is the ball-like area (Fig.S4b), the Si and O peak are much stronger than that of phase 1, representing the aggregation of silicon, oxygen. 